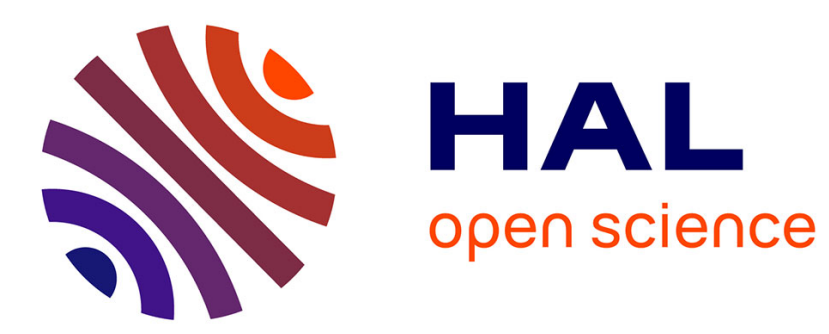

\title{
Practical Asymmetric Synthesis of 1,2-Diamines in the 3-Aminoazepane Series
}

S. Cutri, A. Chiaroni, M. Bonin, L. Micouin, H.-P. Husson

\section{To cite this version:}

S. Cutri, A. Chiaroni, M. Bonin, L. Micouin, H.-P. Husson. Practical Asymmetric Synthesis of 1,2Diamines in the 3-Aminoazepane Series. Journal of Organic Chemistry, 2003, 68 (7), pp.2645-2651. 10.1021/jo026711s . hal-02186903

\section{HAL Id: hal-02186903 https://hal.science/hal-02186903}

Submitted on 17 Jul 2019

HAL is a multi-disciplinary open access archive for the deposit and dissemination of scientific research documents, whether they are published or not. The documents may come from teaching and research institutions in France or abroad, or from public or private research centers.
L'archive ouverte pluridisciplinaire HAL, est destinée au dépôt et à la diffusion de documents scientifiques de niveau recherche, publiés ou non, émanant des établissements d'enseignement et de recherche français ou étrangers, des laboratoires publics ou privés. 


\title{
Practical Asymmetric Synthesis of 1,2-Diamines in the 3-Aminoazepane Series
}

\author{
S. Cutri, A. Chiaroni, M. Bonin*, L. Micouin* and H.-P. Husson \\ Laboratoire de Chimie Thérapeutique, UMR 8638 associée au CNRS et à \\ l'Université René Descartes, Faculté des Sciences Pharmaceutiques et Biologiques \\ 4, av de l'Observatoire, 75270 Paris cedex 06 (France)
}

\begin{abstract}
A. Chiaroni
Institut de Chimie des Substances Naturelles, Avenue de la Terrasse, F-91198 Gif-sur-Yvette (France)
\end{abstract}

\begin{abstract}
A simple and versatile method for the enantio- and diastereoselective synthesis of mono- or disubstituted 3-aminoazepanes is described. The key step involves a highly regioand diastereoselective tandem ring-enlargement/alkylation or reduction process. This novel synthetic route provides enantiomerically pure constrained diamines interesting as scaffolds for medicinal chemistry.
\end{abstract}

\section{Introduction}

Vicinal diamines and their derivatives have been shown to play key roles in medicinal chemistry, coordination chemistry, and asymmetric catalysis. ${ }^{1}$ Among this family, constrained diamine systems continue to attract synthetic interest considering their wide potential as medicinal agents. Novel polyamino derivatives recently reported as potential contrast enhancement agents in MRI have displayed considerable improvement of in vivo stability and biodistribution relative to non-rigid Gadolinium (III) complexes. ${ }^{2}$ Various CNS receptor ligands ${ }^{3}$ and antitumor chiral cis-platin analogues ${ }^{4}$ have been developed in the 3- 
aminoazepane series. Although various synthetic pathways have been devised for the preparation of functionalized azepanes, ${ }^{5}$ a straightforward access to enantiopure polysubstituted 3-aminoazepanes, as general scaffolds for the elaboration of bioactive compounds, would be desirable.

In previous preliminary synthetic studies, we reported the asymmetric synthesis of optically pure trans-2-phenyl- and 2-furyl-3-aminoazepanes 3a,c from 2-cyano-6oxazolopiperidine 1. ${ }^{6}$ The key-step involved a one-pot reduction and ring-enlargement process of intermediates $\mathbf{2}$ occurring in a totally regio- and diastereoselective manner (Scheme 1).

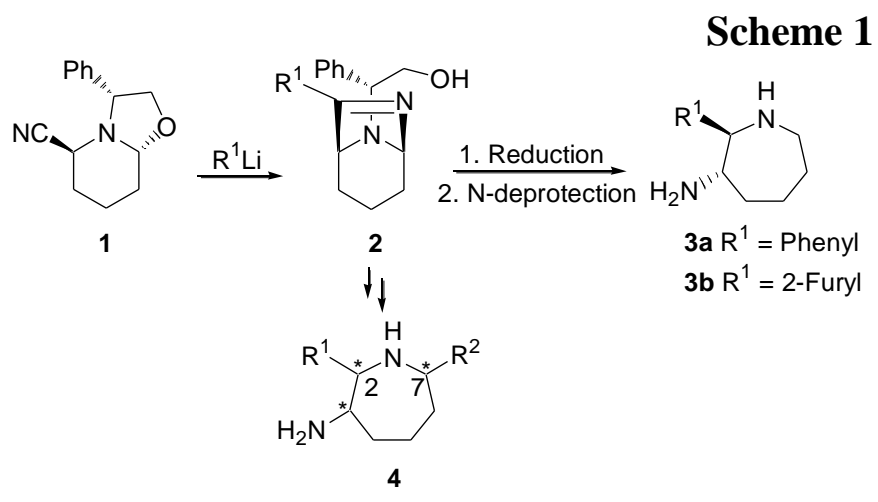

We report in this article our full investigations in this field, as well as the generalization of this original ring-enlargement reaction for the diastereo- and enantioselective preparation of 2,7-disubstituted 3-aminoazepanes 4.

\section{Results and Discussion}

\section{Synthesis of 2-substituted 3-aminoazepanes.}

Multigram quantities of bicyclic imines 2 can be prepared in one step from 2-cyano 6oxazolopiperidine $\mathbf{1}^{7}$ in yields ranging from 74 to $97 \%$ (Scheme 2). 


\section{Scheme $2^{\mathrm{a}}$}

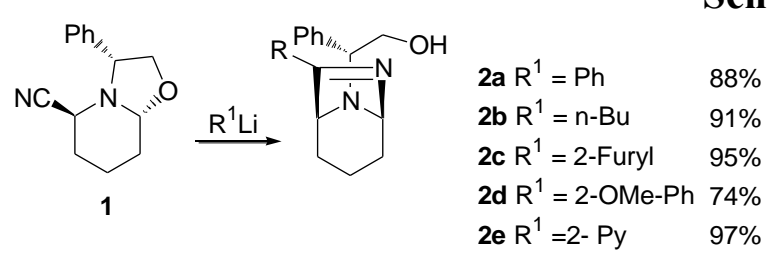

${ }^{\mathrm{a}}$ Reagents and conditions : $\mathrm{R}^{1}=\mathrm{Ph}, \mathrm{n}-\mathrm{Bu}, 2-\mathrm{Py}: \mathrm{R}^{1} \mathrm{Li}, \mathrm{Et}_{2} \mathrm{O},-78^{\circ} \mathrm{C} ; \mathrm{R}^{1}=2-\mathrm{OMe}-\mathrm{Ph}, 2-$

Furyl: $\mathrm{R}^{1} \mathrm{Li}, \mathrm{TMEDA}, \mathrm{THF},-78^{\circ} \mathrm{C}$.

These stable compounds are key intermediates for the ring enlargement strategy. Under protic conditions, reaction of butyl or phenyl derivatives $\mathbf{2 a , b}$ with $\mathrm{NaBH}_{4}$ or $\mathrm{NaBH}_{3} \mathrm{CN}$ have been reported to give piperidines $\mathbf{5}$ or morpholines $\mathbf{6}$ respectively in a high diastereoselective manner (Scheme 3). ${ }^{8}$

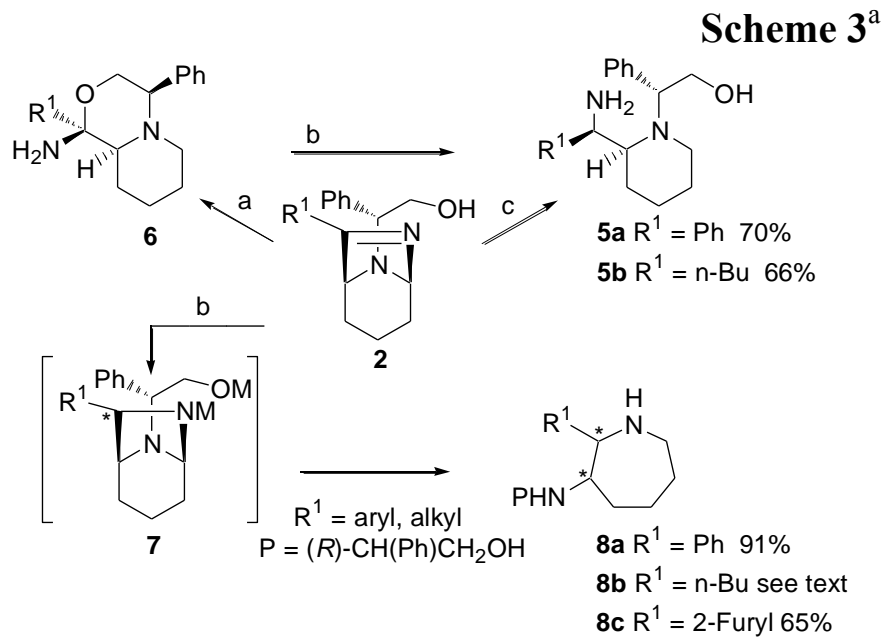

${ }^{a}$ Reagents and conditions : (a) $\mathrm{NaBH}_{3} \mathrm{CN}, \mathrm{H}^{+}$, THF, $\mathrm{MeOH}$; (b) $\mathrm{LAH}, \mathrm{Et}_{2} \mathrm{O}$; (c) $\mathrm{NaBH}_{4}$, $\mathrm{MeOH}$.

Treatment of the same imines with LAH led to a completely different 1,2-diamine system. No trace of six-membered ring derivatives could be observed starting from aromatic conjugated imines 2a,c. However, in the butyl series an inseparable mixture of compounds $\mathbf{8 b}$ and $\mathbf{5 b}$ was obtained. The best conditions were found by adding LAH carefully to an ether solution of the imine $\left(20{ }^{\circ} \mathrm{C}, 3 \mathrm{~h} ; \mathbf{8} \mathbf{b} / \mathbf{5} \mathbf{b}=9 / 1\right)$. The different issues of reductions can be explained by chemoselective nitrogen activation. One the one hand, activation of the 
secondary aminal nitrogen under protic conditions leads to a 6-membered ring, on the other hand activation of the piperidine nitrogen under Lewis acidic conditions leads to the ring enlargement. The lack of selectivity observed in the aliphatic series probably relies on the formation of a transient morpholine $\mathbf{6 b}$, known to be reduced into piperidine systems by LAH. This morpholine was indeed isolated when LAH reduction of $\mathbf{2} \mathbf{b}$ was performed at low temperature $\left(-78{ }^{\circ} \mathrm{C}\right)$.

Since the ring-enlargement proceeds by a two step reduction, isolation of the transient aminal should allow selective functionalization at the C-7 azepane position by a nucleophilic opening with a suitable organometallic nucleophile instead of a hydride. Before selective reductions trials, the alcohol function was first protected in order to avoid the formation of morpholine-type intermediates. Various aryl and alkyl imines 2a-e were thus prepared and protected as O-silylated compounds $\mathbf{1 0 a - e}$ in 70-90\% yield (Scheme 4).

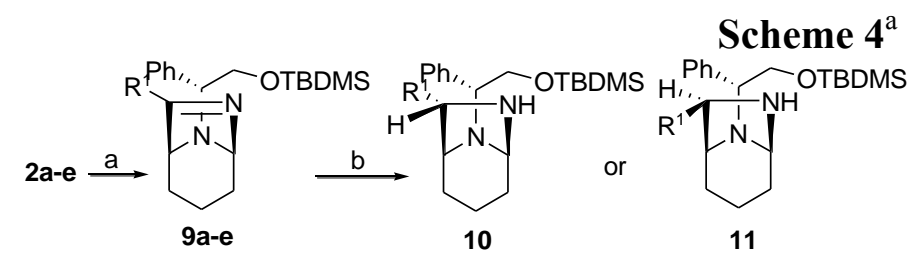

${ }^{a}$ Reagents and conditions : (a) NaH, TBDMSCl, $94 \%(\mathrm{R}=\mathrm{Ph}), 86 \%(\mathrm{R}=\mathrm{n}-\mathrm{Bu}), 81 \%(\mathrm{R}=$ 2-OMe-Ph), $95 \%$ (R = 2-Py); (b) see Table 1 .

Table 1. Diastereoselective access to aminals 10 or 11.

\begin{tabular}{rcccc} 
product $^{\mathrm{a}}$ & $\mathrm{R}^{1}$ & conditions & $\mathbf{1 0 : 1 1}$ & Yield (\%) \\
\hline 11a & $\mathrm{Ph}$ & $\mathrm{Li} / \mathrm{NH}_{3}$ & $19: 81$ & 64 \\
11b & $\mathrm{n}-\mathrm{Bu}$ & $\mathrm{LAH}$ & $<5: 95$ & 65 \\
11d & $2-\mathrm{MeOPh}$ & $\mathrm{LAH}$ & $<5: 95$ & 74 \\
10e & $2-\mathrm{Py}$ & $\mathrm{H}_{2}, \mathrm{Pd} / \mathrm{C}$ & $98: 2$ & 68 \\
\hline
\end{tabular}

${ }^{\mathrm{a}}$ Major diastereomer. ${ }^{\mathrm{b}}$ Yield of diastereomerically pure compound.

Selective imine reductions proved to be particularly troublesome and highly dependent on their substitution patterns. Our first attempts to reduce 9a using LAH under 
various experimental conditions led to either irreproducible results or the known desilylated azepane derivative 8a. More encouraging results were obtained using dissolving-metal conditions. ${ }^{9}$ Treatment of $\mathbf{9 a}$ with lithium in liquid ammonia afforded, in a first experiment, a mixture of aminals 10a and 11a in a $1 / 1$ ratio. Careful optimization of the reprotonation conditions improved this ratio significantly in favor of the 11a isomer, leading to pure material in $64 \%$ yield (Table 1). The presence of a bicyclic aminal could be ascertained by typical NMR chemical shifts (a doublet at $4.58 \mathrm{ppm}$ and a peak at $60.5 \mathrm{ppm}$ observed respectively in the ${ }^{1} \mathrm{H}$ and the ${ }^{13} \mathrm{C}$ NMR spectra were attributed to the $\mathrm{C}-7$ reduced position, two broad deshielded proton signals consistent with the searched bicyclic aminal system). Absolute configuration of the newly created asymmetric center was determined unambiguously by chemical transformation of 11a into O-silylated piperidine derivative 12a by acidic $\mathrm{NaBH}_{3} \mathrm{CN}$ reduction. ${ }^{7}$ In the 2-methoxyphenyl series, simple LAH reduction afforded the corresponding aminal 11d with reasonable yield and high diastereoselectivity, without over-reduction into the corresponding azepane. Both methods failed when applied to the pyridinyl imine 9e reduction. LAH reduction led only to desilylation or degradation material whereas lithium or sodium in liquid ammonia gave an inseparable mixture of aminals 10e and 11e (60:40 ratio, $95 \%$ yield). Pd-catalyzed hydrogenation in various solvents was therefore investigated. The hydrogenation of the imine in ethyl acetate afforded the corresponding aminals 10e and 11e as an equimolar mixture, whereas in methanol, the major derivative 10e could be isolated in a diastereomerically pure form. Finally, 11b could be obtained as a single diastereomer after LAH reduction.

In each case, ${ }^{1} \mathrm{H}$ and ${ }^{13} \mathrm{C}$ NMR spectra revealed characteristic signals of bridged bicyclic structures as shown above. However, the relative configuration of the newly created stereogenic center (C-7) could not be determined directly from the NMR data. This was achieved by correlation with the corresponding erythro $(J=3.9-4.3 \mathrm{~Hz})$ or threo $(J=10.1$ 11.2 Hz) O-silylated aminoalkyl piperidines 12 after acidic $\mathrm{NaBH}_{3} \mathrm{CN}$ aminal reduction of analytical samples (Scheme 5). 


\section{Scheme 5}

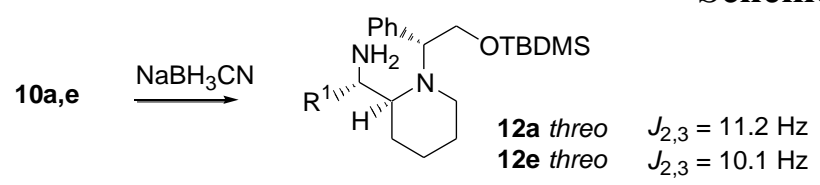

$\mathbf{1 1} \mathbf{a , d} \stackrel{\mathrm{NaBH}_{3} \mathrm{CN}}{\longrightarrow} \mathrm{R}^{1} \stackrel{\mathrm{H}^{\prime \prime \prime}}{\longrightarrow} \begin{array}{ll}\mathbf{1 2 a} \text { erythro } & J_{2,3}=4.3 \mathrm{~Hz} \\ \mathbf{1 2 d} \text { erythro } & J_{2,3}=3.9 \mathrm{~Hz}\end{array}$

Since bicyclic aminals are believed to be intermediates in the one step ring enlargement process, their reduction with LAH should lead to the known azepanes $\mathbf{8}$. This was indeed the case, although this transformation required harsher reaction conditions than with the direct imine reduction. LAH treatment of aminal 11d in refluxing diisopropyl ether afforded in a diastereoselective manner the desilylated and demethylated azepane $\mathbf{8 f}$ with the desired azepane cyclic core $\mathbf{8 d}$ as a minor side product $(\mathbf{8 d} / \mathbf{8 f}=25 / 75){ }^{10}$

\section{Scheme $6^{\mathrm{a}}$}

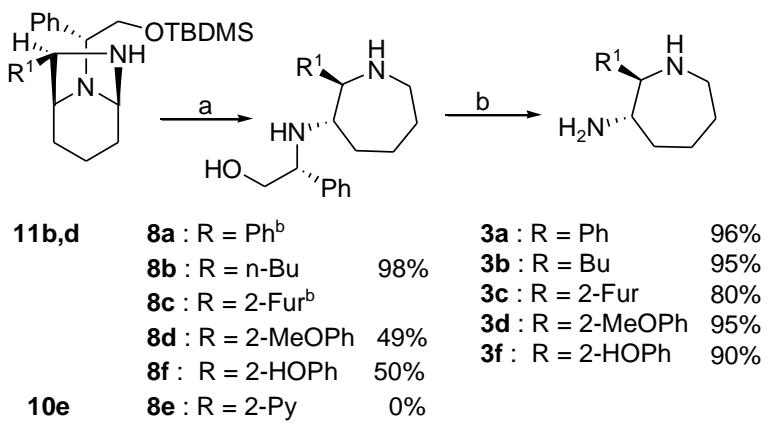

${ }^{a}$ Reagents and conditions : (a) $\mathrm{LAH}, \mathrm{iPr}_{2} \mathrm{O}$ or $\mathrm{Et}_{2} \mathrm{O}$, reflux; (b) $\mathrm{H}_{2}, \mathrm{Pd} / \mathrm{C}, \mathrm{MeOH}-\mathrm{HCl}$ for 8a,b,d,f or $\mathrm{H}_{5} \mathrm{IO}_{6}(2.6 \mathrm{eq}), \mathrm{MeNH}_{2}, \mathrm{H}_{2} \mathrm{O}, \mathrm{MeOH}$, then $\mathrm{MeOH}-\mathrm{HCl}$ for $\mathbf{8 c}$.

${ }^{\mathrm{b}}$ From 2a,c via one step reduction (Scheme 3).

Using milder conditions (refluxing in diethyl ether), the desired product 8d was obtained as the major product $(\mathbf{8 d} / \mathbf{8 f}=80 / 20)$. Unfortunately, presence of a pyridine ring seemed to inhibit the ring-enlargement process. Finally, unprotected 2-substituted 3-amino 
azepanes were obtained in good yield after hydrogenolysis or oxidative cleavage. Interestingly, using this two step process, diamine $\mathbf{8 b}$ could be obtained in good yield and total diastereoselectivity, without any 6-membered ring contaminants. Once again, the trans relative configuration of such azepanes could be ascertain by the typical $\mathrm{H}-2 / \mathrm{H}-3$ coupling constant of $9 \mathrm{~Hz}$.

\section{Synthesis of 2,7-disubstituted 3-aminoazepanes.}

During the ring enlargement process, a transient seven-membered imine was reduced by an hydride. However, the use a of Lewis acidic nucleophile should lead to the same reactive species from bicyclic aminals $\mathbf{1 0}$ or $\mathbf{1 1}$ and enable further functionalizations of the azepane at the C-7 position. Although the reactivity of such aminals is unknown, the related reaction of nucleophilic reagents, especially organomagnesium compounds, with oxazolidines is well documented. ${ }^{11}$

\section{Scheme 7}

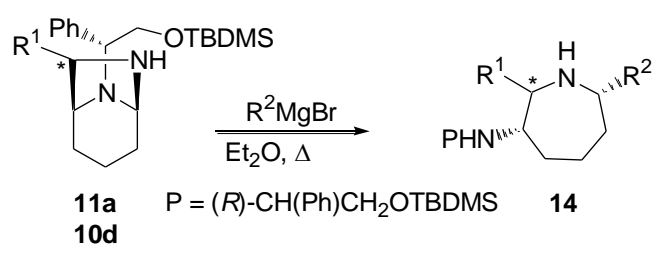

Table 2. Diastereoselective access to polysubstituted azepanes 14

\begin{tabular}{rcccc} 
product & $\mathrm{R}^{1}$ & $\mathrm{R}^{2}$ & Yield $(\%)^{\mathrm{a}}$ & d.e. $(\%)^{\mathrm{b}}$ \\
\hline $\mathbf{1 4 a}$ & $\mathrm{Ph}(2 R)$ & $\mathrm{Me}$ & 57 & $>95$ \\
$\mathbf{1 4 b}$ & $\mathrm{Ph}(2 R)$ & $i$ - $\mathrm{Bu}$ & 84 & $>95$ \\
$\mathbf{1 4 c}$ & $\mathrm{Ph}(2 R)$ & $\mathrm{Bn}$ & 95 & $>95$ \\
$\mathbf{1 4 d}$ & $2-\mathrm{Py}(2 R)$ & $\mathrm{Me}$ & 69 & $>95$ \\
$\mathbf{1 4 e}$ & $2-\mathrm{Py}(2 R)$ & $i$ - $\mathrm{Bu}$ & 70 & $>95$ \\
$\mathbf{1 4 f}$ & $2-\mathrm{Py}(2 R)$ & $\mathrm{Bn}$ & 65 & $>95$ \\
\hline
\end{tabular}

${ }^{a}$ Yield of diastereomerically pure compound.

${ }^{b}$ Determined by ${ }^{1} \mathrm{H}$ NMR of the crude reaction mixture. 
Initial experiments were performed on phenylaminal 11a, using methylmagnesium bromide as a nucleophile and a Lewis acid (Table 2). The best results were obtained via refluxing 11a in ether with an excess of organomagnesium reagent (3.5 equiv.), leading to compound 14a in $57 \%$ yield as a single diastereomer. The formation of a ring-enlarged derivative could be ascertained by typical NMR data : ${ }^{13} \mathrm{C}$ NMR spectrum displayed the three $\mathrm{CH}$ peaks at $49.5,62.2,62.8 \mathrm{ppm}$ and one methyl signal at $23.4 \mathrm{ppm}$ as expected from the alkylation process. The ${ }^{1} \mathrm{H}$ NMR spectrum showed a multiplet at $2.90 \mathrm{ppm}$ for the $\mathrm{C}-7$ proton and a doublet at $\delta 3.82 \mathrm{ppm}$ for the $\mathrm{C}-2$ proton.

The use of iso-butyl as well as benzyl Grignards as nucleophiles afforded exclusively ring-enlarged derivatives $\mathbf{1 4 b , c}$ in diastereomerically pure form. Interestingly, no reduction product arising from an hydride transfer with the branched organomagnesium reagent could be detected. Unlike the LAH reduction of aminals $\mathbf{1 1 b}, \mathbf{d}$, the ring-enlargement occurred without any O-desilylation. Final compounds $\mathbf{4 a , b , c}$ could be obtained after desilylation and hydrogenolysis.

\section{Scheme 8}

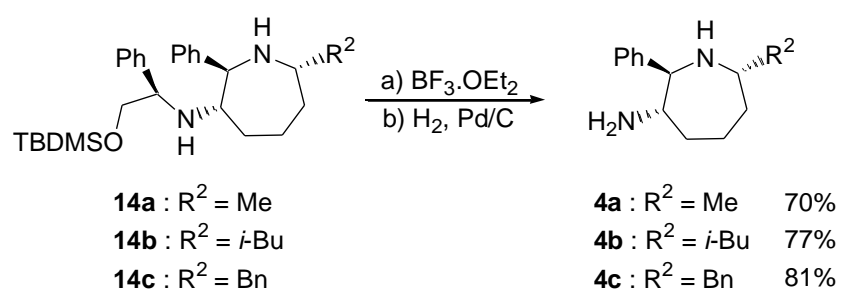

Following standard procedure, benzylazepane $4 \mathbf{c}$ was finally converted to crystalline benzamide 15, and its relative configuration was determined by X-ray crystallography analysis. The 3,7-cis configuration could be attributed to derivatives $\mathbf{4 a , b}$ by comparisons of their ${ }^{1} \mathrm{H}$ and ${ }^{13} \mathrm{C}$ NMR spectra. 


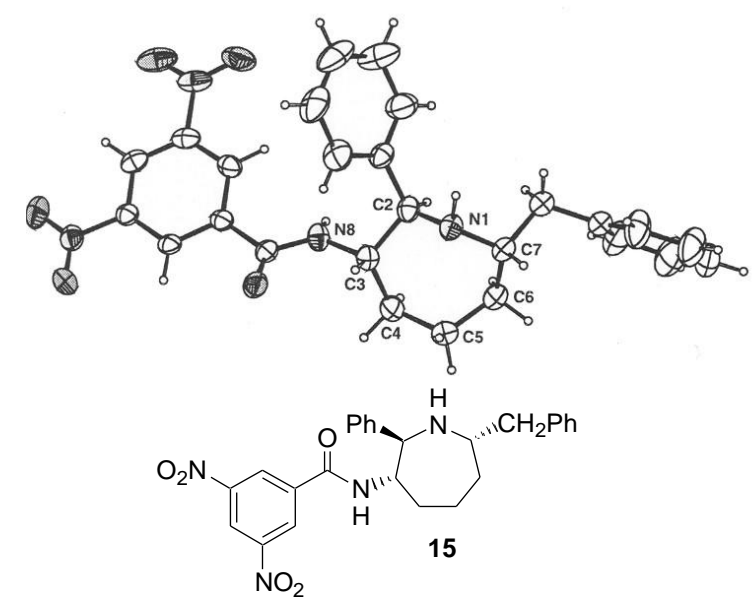

Figure 1 : X-ray structure of derivative 15.

The same ring-enlargement reactions were then performed starting from pyridyl aminal 10e, in order to study the influence of each stereogenic center on the stereochemical outcome of this reaction. Compounds 14d,e,f were obtained in good yields as single diastereomers. Once again, a 3,7-cis configuration could be established for compound $\mathbf{1 4 f}$ by n.O.e experiments on its desilylated derivative, and attributed to the other compounds by comparisons of their ${ }^{1} \mathrm{H}$ and ${ }^{13} \mathrm{C}$ NMR spectra.

Alkylations are therefore totally diastereoselective. Surprisingly, the stereochemical outcome is independent of the $\mathrm{C}-2$ stereogenic center configuration, unlike previous examples in the piperidine series. ${ }^{12}$ The 3,7-cis configuration could be explained by a mechanism involving an intramolecular delivery of nucleophile in an early transition state (Figure 2).

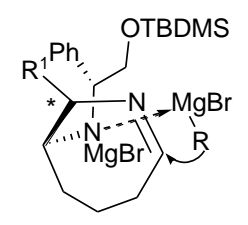

Figure 2 : Origin of the relative configuration of disubstituted aminoazepanes. 
In summary, we have developed a simple and versatile method for the rapid elaboration of 2-mono- or 2,7-disubstituted 3-aminoazepanes. We have shown that bicyclic aminals are powerful synthetic intermediates in allowing a tandem ringenlargement/alkylation process in a highly regio- and diastereoselective manner. This novel synthetic route leads to various enantiomerically constrained diamines interesting as scaffolds for medicinal chemistry. Application of this method to the preparation of biologically active compounds is currently underway in our laboratory.

\section{Experimental section}

General Methods. ${ }^{1} \mathrm{H}$ NMR (300 or $\left.400 \mathrm{MHz}\right)$ and ${ }^{13} \mathrm{C}$ NMR $(75 \mathrm{MHz})$ were recorded in $\mathrm{CDCl}_{3}$ or $\mathrm{CD}_{3} \mathrm{OD}$ solution. IR spectra were recorded as thin film unless otherwise stated. Mass spectral data were recorded in chemical-ionization (CI) mode. Tetrahydrofuran (THF) and ether $\left(\mathrm{Et}_{2} \mathrm{O}\right.$ or $\left.\mathrm{iPr}_{2} \mathrm{O}\right)$ were distilled from sodium/benzophenone immediately prior to use; $\mathrm{CH}_{2} \mathrm{Cl}_{2}, \mathrm{TMEDA}, \mathrm{Et}_{3} \mathrm{~N}, \mathrm{MeOH}, \mathrm{CHCl}_{3}$ were distilled from $\mathrm{CaH}_{2}$. Compounds 1, 2a, 2b, 3a, 3c were prepared according to previously reported procedures. ${ }^{6,7,8}$

$(1 S, \quad 5 S) \quad 2-[7-(2-M e t h o x y p h e n y l)-6,8-d i a z a b i c y c l o[3.2 .1]$ oct-6-en-8-yl]-(2R)-phenylethanol 2 d. Anisole $(4.28 \mathrm{~mL}, 39.5 \mathrm{mmol})$ was added to a cold $\left(0{ }^{\circ} \mathrm{C}\right)$ mixture of $n$ butyllithium (1.6 $\mathrm{M}$ in hexanes, $16.4 \mathrm{~mL}, 26 \mathrm{mmol})$ and TMEDA $(3.96 \mathrm{~mL}, 26.3 \mathrm{mmol})$ in THF (20 mL) under an atmosphere of Ar. The reaction mixture was maintained at $20^{\circ} \mathrm{C}$ for $45 \mathrm{~min}$ and then cooled to $-78{ }^{\circ} \mathrm{C}$. A solution of 2-cyano-6-phenyloxazolopiperidine (1) (2.0 $\mathrm{g}, 8.8 \mathrm{mmol})$ in THF $(20 \mathrm{~mL})$ was added and the reaction mixture was allowed to warm to room temperature and maintained overnight. The reaction mixture was cautiously diluted with a saturated, aqueous solution of $\mathrm{NH}_{4} \mathrm{Cl}$, extracted with $\mathrm{CH}_{2} \mathrm{Cl}_{2}(2 \mathrm{x})$, and the combined organic layers were dried $\left(\mathrm{MgSO}_{4}\right)$. The solvent was evaporated to give an oily residue. The 
residue was purified by chromatography $\left(\mathrm{CH}_{2} \mathrm{Cl}_{2} / \mathrm{MeOH} 95 / 5\right)$ to provide imine $\mathbf{2 d}$ (yellow oil, $2.18 \mathrm{~g}, 74 \%) .[\alpha] \mathrm{D}=+46\left(c \quad 1.4, \mathrm{CH}_{2} \mathrm{Cl}_{2}\right)$; IR (neat) $3326,2940,1656,1599 \mathrm{~cm}^{-1}$; MS $m / z 337\left(\mathrm{MH}^{+}\right) ;{ }^{1} \mathrm{H}$ NMR $\left(\mathrm{CDCl}_{3}\right) \delta$ 1.20-1.80 (m, 6H), $3.52(\mathrm{t}, J=4.8 \mathrm{~Hz}, 1 \mathrm{H}), 3.53(\mathrm{~s}, 3 \mathrm{H})$, $3.77(\mathrm{dd}, J=11.1,4.3 \mathrm{~Hz}, 1 \mathrm{H}), 3.87$ (dd, $J=11.1,5.2 \mathrm{~Hz}, 1 \mathrm{H}), 4.22$ (t, $J=2.9 \mathrm{~Hz}, 1 \mathrm{H}), 5.45$ (t, $J=2.6 \mathrm{~Hz}, 1 \mathrm{H}), 6.83(\mathrm{~d}, J=7.4 \mathrm{~Hz}, 1 \mathrm{H}), 6.94(\mathrm{td}, J=7.7,0.9 \mathrm{~Hz}, 1 \mathrm{H}), 7.21-7.33(\mathrm{~m}, 5 \mathrm{H})$, $7.37(\mathrm{td}, J=7.7,1.7 \mathrm{~Hz}, 1 \mathrm{H}), 7.79(\mathrm{dd}, J=7.7,1.8 \mathrm{~Hz}, 1 \mathrm{H}) ;{ }^{13} \mathrm{C} \mathrm{NMR}\left(\mathrm{CDCl}_{3}\right) \delta$ 17.0, 24.4, 26.0, 55.0, 65.1, 65.9, 68.0, 84.3, 111.2, 120.7, 121.6, 127.4, 128.3, 128.5, 130.6, 132.1, $141.1,158.6,172.2$.

$(1 S, \quad 5 S) \quad$ 2-(7-Furan-2-yl-6,8-diazabicyclo[3.2.1]oct-6-en-8-yl)-(2R)-phenyl-ethanol 2c (yellow oil, $2.47 \mathrm{~g}, 95 \%$ ). [ $\alpha] \mathrm{D}=-11\left(\mathrm{c} 1.1, \mathrm{CH}_{2} \mathrm{Cl}_{2}\right)$; IR (neat) 3320, 2947, $1622 \mathrm{~cm}^{-1}$; MS $m / z 297\left(\mathrm{MH}^{+}\right) ;{ }^{1} \mathrm{H}$ NMR $\left(\mathrm{CDCl}_{3}\right) \delta 1.20-1.85(\mathrm{~m}, 6 \mathrm{H}), 3.47(\mathrm{t}, J=4.7 \mathrm{~Hz}, 1 \mathrm{H}), 3.78(\mathrm{dd}, J$ $=11.1,4.2 \mathrm{~Hz}, 1 \mathrm{H}), 3.85(\mathrm{t}, J=2.9 \mathrm{~Hz}, 1 \mathrm{H}), 3.92(\mathrm{dd}, J=11.1,5.2 \mathrm{~Hz}, 1 \mathrm{H}), 5.50(\mathrm{t}, J=2.9$ $\mathrm{Hz}, 1 \mathrm{H}), 6.46(\mathrm{dd}, J=3.4,1.5 \mathrm{~Hz}, 1 \mathrm{H}), 6.67(\mathrm{~d}, J=3.4 \mathrm{~Hz}, 1 \mathrm{H}), 7.25-7.35(\mathrm{~m}, 5 \mathrm{H}), 7.51$ (t, $J=1.5 \mathrm{~Hz}, 1 \mathrm{H}) ;{ }^{13} \mathrm{C} \mathrm{NMR}\left(\mathrm{CDCl}_{3}\right) \delta 16.7,25.3,25.4,64.6,65.8,66.3,86.2,111.9,114.4$, $127.8,128.4,128.6,140.2,145.4,162.4$.

(1S, 5S) 2R-Phenyl-2-(7-pyridin-2-yl-6,8-diazabicyclo[3.2.1]oct-6-en-8-yl)-ethanol 2e. $n$ BuLi (21.9 mL, 1.6 M in hexanes, $35.1 \mathrm{mmol}$ ) was added to a stirred solution of 2bromopyridine $(4.48 \mathrm{~mL}, 46.8 \mathrm{mmol})$ in anhydrous $\mathrm{Et}_{2} \mathrm{O}(30 \mathrm{~mL})$ at $-70{ }^{\circ} \mathrm{C}$ under argon. After $30 \mathrm{~min}$ a solution of 2-cyano-6-phenyl-oxazolopiperidine 1 (2 g, $8.77 \mathrm{mmol})$ in $\mathrm{Et}_{2} \mathrm{O}$ $(10 \mathrm{~mL})$ was added. The mixture was stirred at $-70{ }^{\circ} \mathrm{C}$ for $5 \mathrm{~h}$. The reaction mixture was cautiously diluted with a saturated, aqueous solution of $\mathrm{NH}_{4} \mathrm{Cl}$, extracted with $\mathrm{CH}_{2} \mathrm{Cl}_{2}(2 \mathrm{x})$, and the combined organic layers were dried $\left(\mathrm{MgSO}_{4}\right)$. The residue was purified by chromatography $\left(\mathrm{CH}_{2} \mathrm{Cl}_{2} / \mathrm{MeOH} 95 / 5\right)$ to provide $2 \mathrm{c}$ (amorphous yellow solid, $2.61 \mathrm{~g}, 97 \%$ ). $[\alpha] \mathrm{D}=-44\left(c 1.1, \mathrm{CH}_{2} \mathrm{Cl}_{2}\right)$; IR (neat) 3330, 2945, 2871, 1609, $1586 \mathrm{~cm}^{-1} ; \mathrm{MS} \mathrm{m} / z 308$ $\left(\mathrm{MH}^{+}\right) ;{ }^{1} \mathrm{H} \mathrm{NMR}\left(\mathrm{CDCl}_{3}\right) \delta 1.13-1.92(\mathrm{~m}, 6 \mathrm{H}), 3.57(\mathrm{t}, J=5.4 \mathrm{~Hz}, 1 \mathrm{H}), 3.85(\mathrm{~d}, J=5.4 \mathrm{~Hz}$, 
$1 \mathrm{H}), 4.38(\mathrm{t}, J=2.9 \mathrm{~Hz}, 1 \mathrm{H}), 5.49(\mathrm{t}, J=2.5 \mathrm{~Hz}, 1 \mathrm{H}), 7.13-7.24(\mathrm{~m}, 5 \mathrm{H}), 7.29$ (ddd, $J=7.7$, 4.8, 1.5 Hz, 1H), 7.68 (td, $J=7.7,1.5 \mathrm{~Hz}, 1 \mathrm{H}$ ), 7.96 (br. d, $J=7.7 \mathrm{~Hz}, 1 \mathrm{H}$ ), 8.53 (br. d, $J=4.8$ $\mathrm{Hz}, 1 \mathrm{H}) ;{ }^{13} \mathrm{C} \mathrm{NMR}\left(\mathrm{CDCl}_{3}\right) \delta 17.0,24.7,25.1,64.0,65.1,66.4,87.2,122.1,124.9,127.6$, $128.2,128.4,136.3,139.8,149.2,151.3,173.2$.

\section{General procedure for the preparation of $O$-silylated imines 9.}

The preparation of imine 9a is representative. $\mathrm{NaH}(60 \%$ suspension in mineral oil, $614 \mathrm{mg}$, $15.4 \mathrm{mmol})$ was added at $0{ }^{\circ} \mathrm{C}$ under argon to a stirred solution of imine $2 \mathrm{a}(2.36 \mathrm{~g}, 7.71$ $\mathrm{mmol})$ in THF (94 mL). After $30 \mathrm{~min}$ at room temperature, ter-butyldimethylsilyl chloride ( $2.33 \mathrm{~g}, 15.4 \mathrm{mmol})$ was added. The resulting mixture was stirred for an additional $16 \mathrm{~h}$ at 20 ${ }^{\circ} \mathrm{C}$. The reaction mixture was cautiously diluted with a saturated, aqueous solution of $\mathrm{NH}_{4} \mathrm{Cl}$, extracted with $\mathrm{CH}_{2} \mathrm{Cl}_{2}(2 \mathrm{x})$, and the combined organic layers were dried $\left(\mathrm{MgSO}_{4}\right)$. The residue was purified by flash chromatography (Cyclohexane/AcOEt 1/1, then 2/8, then AcOEt) to provide 9a as a yellow oil (3.04 g, 94\%).

$(1 S, \quad 5 S) \quad 8$-[2-(tert-Butyl-dimethyl-silanyloxy)-(1R)-phenyl-ethyl]-7-phenyl-6,8-diazabicyclo[3.2.1] oct-6-ene 9a. Oil, $[\alpha] \mathrm{D}=+10\left(c 0.9, \mathrm{CH}_{2} \mathrm{Cl}_{2}\right)$; IR (neat) 2951, 2858, $1605 \mathrm{~cm}$ '; MS m/z $421\left(\mathrm{MH}^{+}\right)$; ${ }^{1} \mathrm{H}$ NMR $\left(\mathrm{CDCl}_{3}\right) \delta-0.22$ (s, 3H), -0.17 (s, 3H), 0.71 (s, 9H), 1.11$1.78(\mathrm{~m}, 6 \mathrm{H}), 3.32(\mathrm{t}, J=5.6 \mathrm{~Hz}, 1 \mathrm{H}), 3.63(\mathrm{dd}, J=10.3,5.6 \mathrm{~Hz}, 1 \mathrm{H}), 3.82(\mathrm{t}, J=2.9 \mathrm{~Hz}, 1 \mathrm{H})$, $3.88(\mathrm{dd}, J=10.3,5.6 \mathrm{~Hz}, 1 \mathrm{H}), 5.54(\mathrm{t}, J=2.7 \mathrm{~Hz}, 1 \mathrm{H}), 7.14-7.37(\mathrm{~m}, 8 \mathrm{H}), 7.61(\mathrm{~m}, 2 \mathrm{H}) ;{ }^{13} \mathrm{C}$ NMR $\left(\mathrm{CDCl}_{3}\right) \delta-1.5,-0.5,16.9,24.8,18.2,25.9,66.5,68.0,86.0,127.4,128.0,128.4$, $128.7,130.9,132.5,142.8,150.2,171.8$.

(1S, 5S)7-Butyl-8-[2-(tert-butyl-dimethyl-silanyloxy)-(1R)-phenyl-ethyl]-6,8-diazabicyclo [3.2.1]oct-6-ene 9b (yellow oil, $2.65 \mathrm{~g}, 86 \%$ ). [ $\alpha] \mathrm{D}=-18\left(c 1.8, \mathrm{CH}_{2} \mathrm{Cl}_{2}\right)$; IR (neat) 2954, 2856, $1636 \mathrm{~cm}^{-1}$; MS m/z $401\left(\mathrm{MH}^{+}\right) ;{ }^{1} \mathrm{H} \mathrm{NMR}\left(\mathrm{CDCl}_{3}\right) \delta-0.26(\mathrm{~s}, 3 \mathrm{H}), 0.20,(\mathrm{~s}, 3 \mathrm{H}), 0.70$ (s, 9H), 0.83 (t, $J=7.3 \mathrm{~Hz}, 1 \mathrm{H}), 1.10-1.80(\mathrm{~m}, 10 \mathrm{H}), 2.13(\mathrm{~m}, 1 \mathrm{H}), 2.27(\mathrm{~m}, 1 \mathrm{H}), 3.24(\mathrm{~m}$, 2H), 3.57 (dd, $J=10.2,6.0 \mathrm{~Hz}, 1 \mathrm{H}), 3.84$ (dd, $J=10.2,5.4 \mathrm{~Hz}, 1 \mathrm{H}), 5.27$ (br.s, 1H), 7.02- 
$7.36(\mathrm{~m}, 5 \mathrm{H}) ;{ }^{13} \mathrm{C} \mathrm{NMR}\left(\mathrm{CDCl}_{3}\right) \delta-5.3,14.1,18.3,18.5,23.0,24.4,25.2,26.1,28.4,31.2$, $66.8,68.2,68.5,85.4,127.5,128.5,128.8,128.9,142.1,177.2$.

$(1 S, 5 S)$ 8-[2-(tert-Butyl-dimethyl-silanyloxy)-(1R)-phenyl-ethyl]-7-(2-methoxy-phenyl)6,8-diazabicyclo[3.2.1]oct-6-ene 9d (yellow oil, $2.81 \mathrm{~g}, 81 \%$ ). $[\alpha] \mathrm{D}=+72\left(c 1.0, \mathrm{CH}_{2} \mathrm{Cl}_{2}\right)$; IR (neat) 2951, 2855, $1600 \mathrm{~cm}^{-1}$; MS m/z $451\left(\mathrm{MH}^{+}\right) ;{ }^{1} \mathrm{H}$ NMR $\left(\mathrm{CDCl}_{3}\right) \delta-0.17(\mathrm{~s}, 3 \mathrm{H})$, $0.10(\mathrm{~s}, 3 \mathrm{H}), 0.77(\mathrm{~s}, 9 \mathrm{H}), 1.20-1.90(\mathrm{~m}, 6 \mathrm{H}), 3.47(\mathrm{t}, J=5.6 \mathrm{~Hz}, 1 \mathrm{H}), 3.53(\mathrm{~s}, 3 \mathrm{H}), 3.68(\mathrm{dd}, J$ $=10.2,5.8 \mathrm{~Hz}, 1 \mathrm{H}), 3.94(\mathrm{dd}, J=10.2,5.4 \mathrm{~Hz}, 1 \mathrm{H}), 4.13(\mathrm{t}, J=3.5 \mathrm{~Hz}, 1 \mathrm{H}), 5.56$ (t, $J=2.6$ $\mathrm{Hz}, 1 \mathrm{H}), 6.81$ (d, $J=8.0 \mathrm{~Hz}, 1 \mathrm{H}), 6.96(\mathrm{td}, J=7.5,0.9 \mathrm{~Hz}, 1 \mathrm{H}), 7.16-7.37$ (m, 5H), $7.86(\mathrm{dd}$, $J=7.7,1.8 \mathrm{~Hz}, 1 \mathrm{H}) ;{ }^{13} \mathrm{C} \mathrm{NMR}\left(\mathrm{CDCl}_{3}\right) \delta-1.5,17.0,18.3,24.3,25.9,54.9,66.4,68.3,68.8$, $84.5,111.3,120.7,122.4,127.0,128.0,128.8,130.4,131.8,142.4,158.8,171.9$.

$(1 S, 5 S)$ 8-[2-(tert-Butyl-dimethyl-silanyloxy)-1R-phenyl-ethyl]-7-pyridin-2-yl-6,8-diazabicyclo[3.2.1]oct-6-ene 9e (yellow oil, $3.08 \mathrm{~g}, 95 \%$ ). [ $\alpha] \mathrm{D}=-1$ (c 1.4, $\mathrm{CH}_{2} \mathrm{Cl}_{2}$ ); IR (neat) 2950, 2927, 2855, $1607 \mathrm{~cm}^{-1}$; MS m/z $422\left(\mathrm{MH}^{+}\right) ;{ }^{1} \mathrm{H}$ NMR $\left(\mathrm{CDCl}_{3}\right) \delta-0.19(\mathrm{~s}, 3 \mathrm{H}),-0.16(\mathrm{~s}$, $3 \mathrm{H}), 0.75(\mathrm{~s}, 9 \mathrm{H}), 1.06-1.85(\mathrm{~m}, 6 \mathrm{H}), 3.37(\mathrm{t}, J=5.6 \mathrm{~Hz}, 1 \mathrm{H}), 3.64(\mathrm{dd}, J=10.3,5.6 \mathrm{~Hz}, 1 \mathrm{H})$, $3.90(\mathrm{dd}, J=10.3,5.6 \mathrm{~Hz}, 1 \mathrm{H}), 4.13(\mathrm{t}, J=2.9 \mathrm{~Hz}, 1 \mathrm{H}), 5.67$ (t, $J=2.5 \mathrm{~Hz}, 1 \mathrm{H}), 7.12-7.26$ (m, $6 \mathrm{H}), 7.88(\mathrm{td}, J=7.8,1.7 \mathrm{~Hz}, 1 \mathrm{H}), 8.10(\mathrm{~d}, J=7.8 \mathrm{~Hz}, 1 \mathrm{H}), 8.48(\mathrm{dt}, J=4.8,0.9 \mathrm{~Hz}, 1 \mathrm{H}) ;{ }^{13} \mathrm{C}$ $\operatorname{NMR}\left(\mathrm{CDCl}_{3}\right) \delta-1.5,17.0,18.2,24.4,24.6,25.9,66.4,66.9,68.1,86.3,122.3,124.9,127.2$, $128.3,128.5,136.4,140.6,149.4,152.0,173.3$.

Preparation of aminal 11a. $\mathrm{NH}_{3}(3.5 \mathrm{~mL})$ was condensed at $-78{ }^{\circ} \mathrm{C}$ under argon onto a stirred solution of $9 \mathrm{a}(500 \mathrm{mg}, 1.19 \mathrm{mmol})$ in THF $(2.5 \mathrm{~mL})$. After addition of small pieces of lithium (25 mg, $3.56 \mathrm{mmol})$, stirring was continued for $3 \mathrm{~h}$. A solution of anhydrous tertbutanol $(527 \mathrm{mg}, 7.13 \mathrm{mmol})$ in THF $(10 \mathrm{~mL})$ was then added dropwise at $-40{ }^{\circ} \mathrm{C}$. The reaction mixture was allowed to warm gradually to ambient temperature with stirring for the slow evaporation of $\mathrm{NH}_{3}$. After addition of solid $\mathrm{NH}_{4} \mathrm{Cl}(763 \mathrm{mg}, 14.3 \mathrm{mmol})$, the reaction 
mixture was filtered through a celite bed, the organic layers were dried over $\mathrm{MgSO}_{4}$ and concentrated to give a mixture of diastereomers $(\mathbf{1 1 a} / \mathbf{1 0 a}, 81 / 19)$. The residue was purified by chromatography $\left(\mathrm{AcOEt} / \mathrm{MeCN} / \mathrm{NH}_{4} \mathrm{OH}, 246 / 3 / 1\right)$ to provide the major compound 11a as a yellow oil (321 mg, 64\%).

$(1 S, \quad 5 S) \quad 8$-[2-(tert-Butyl-dimethyl-silanyloxy)-(1R)-phenyl-ethyl]-7R-phenyl-6,8-diaza bicyclo [3.2.1]octane 11a. Oil. [ $\alpha$ ] $\mathrm{D}=-5\left(c 1.0, \mathrm{CH}_{2} \mathrm{Cl}_{2}\right)$; IR (neat) 3427, 2928, 2855, 1493 $\mathrm{cm}^{-1}$; MS m/z $423\left(\mathrm{MH}^{+}\right) ;{ }^{1} \mathrm{H}$ NMR $\left(\mathrm{CDCl}_{3}\right) \delta-0.20(\mathrm{~s}, 3 \mathrm{H}),-0.16(\mathrm{~s}, 3 \mathrm{H}), 0.72(\mathrm{~s}, 9 \mathrm{H}), 0.98-$ 1.79 (m, 6H), 2.53 (br. s, 1H), 3.19 (br. s, 1H), 3.59 (m, 2H), 3.88 (dd, $J=11.7,7.6 \mathrm{~Hz}, 1 \mathrm{H})$, 4.58 (d, $J=5.8 \mathrm{~Hz}, 1 \mathrm{H}), 4.74$ (br. s, $1 \mathrm{H}), 7.0-7.34$ (m, 10H); ${ }^{13} \mathrm{C} \mathrm{NMR}\left(\mathrm{CDCl}_{3}\right) \delta-1.4,-1.5$, $16.4,18.3,26.0,26.8,33.5,60.7,62.0,65.9,69.2,74.7,125.8,127.1,127.5,127.8,128.4$, 128.6, 141.6, 142.1.

\section{Preparation of aminal 11b,d.}

The preparation of aminal 11d is representative. To a solution of imine 9d (500 $\mathrm{mg}, 1.11$ $\mathrm{mmol})$ in $\mathrm{Et}_{2} \mathrm{O}(25 \mathrm{~mL})$ under argon was carefully added $\mathrm{LiAlH}_{4}(329 \mathrm{mg}, 8.67 \mathrm{mmol})$. The reaction mixture was stirred $3 \mathrm{~h}$ at room temperature then treated successively with aqueous $\mathrm{NaOH}(1 \mathrm{~N}, 658 \mu \mathrm{L})$ and $\mathrm{H}_{2} \mathrm{O}(987 \mu \mathrm{L})$. After filtration through celite, the organic phase was concentrated in vacuo. The residue was purified by flash chromatography (AcOEt/MeCN/NH $4 \mathrm{OH}, 237 / 12 / 1)$ to provide 11d as a colorless oil (371 mg, 74\%).

$(1 S, \quad 5 S) \quad 8$-[2-(tert-Butyl-dimethyl-silanyloxy)-(1R)-phenyl-ethyl]-(7R)-(2-methoxy phenyl)-6,8-diaza-bicyclo[3.2.1]octane 11d. Oil. $[\alpha] \mathrm{D}=+12\left(\right.$ c $\left.0.8, \mathrm{CH}_{2} \mathrm{Cl}_{2}\right)$; IR (neat) 3320, 2952, 2926, $1492 \mathrm{~cm}^{-1}$; MS m/z $453\left(\mathrm{MH}^{+}\right)$; ${ }^{1} \mathrm{H}$ NMR $\left(\mathrm{CDCl}_{3}\right) \delta-0.17$ (s, 3H), -0.12 (s, 3H), 0.74 (s, 9H), 0.80-1.78 (m, 6H), 2.43 (s, 1H), 3.43 (br. s, 3H), 3.36-3.47 (m, 1H), 3.603.73 (m, 2H), 3.93 (br.s, 1H), 4.58 (d, $J=4.1 \mathrm{~Hz}, 1 \mathrm{H}), 4.67$ (br.s, 1H), 6.63 (d, $J=7.3 \mathrm{~Hz}$, $1 \mathrm{H}), 6.86(\mathrm{t}, J=7.3 \mathrm{~Hz}, 1 \mathrm{H}), 7.08(\mathrm{td}, J=7.6,1.4 \mathrm{~Hz}, 1 \mathrm{H}), 7.17-7.38(\mathrm{~m}, 5 \mathrm{H}), 7.97$ (d, $J=7.9$ $\mathrm{Hz}, 1 \mathrm{H}) ;{ }^{13} \mathrm{C} \mathrm{NMR}\left(\mathrm{CDCl}_{3}\right) \delta-1.4,-1.5,16.3,18.3,25.9,29.8,33.4,54.9,57.8,60.3,66.6$, $69.1,74.3,109.5,120.0,127.0,127.3,128.1,128.5,128.8,130.2,141.7,156.6$. 


\section{(1S, $5 S) \quad 7 R$-Butyl-8-[2-(tert-butyl-dimethyl-silanyloxy)-(1R)-phenyl-ethyl]-6,8-diaza-} bicyclo[3.2.1] octane 11b (colorless oil, $290 \mathrm{mg}, 65 \%$ ). [ $\alpha] \mathrm{D}=+6\left(c 1.0, \mathrm{CH}_{2} \mathrm{Cl}_{2}\right.$ ); IR (neat) 2954, 2929, 2857, $1463 \mathrm{~cm}^{-1}$; MS m/z $403\left(\mathrm{MH}^{+}\right) ;{ }^{1} \mathrm{H}$ NMR $\left(\mathrm{CDCl}_{3}\right) \delta-0.22(\mathrm{~s}, 3 \mathrm{H}),-0.17$ (s, 3H), $0.72(\mathrm{~s}, 9 \mathrm{H}), 0.81$ (t, $J=7.1 \mathrm{~Hz}, 1 \mathrm{H}), 0.96-1.91(\mathrm{~m}, 12 \mathrm{H}), 2.76$ (br.s, 1H), 2.80 (br.s, 1H), 3.22 (dt, $J=8.7,5.3 \mathrm{~Hz}, 1 \mathrm{H}), 3.55(\mathrm{~m}, 2 \mathrm{H}), 3.82$ (dd, $J=12.2,7.7 \mathrm{~Hz}, 1 \mathrm{H}), 4.56$ (br.s, 1H), 7.11-7.37 (m, 5H); ${ }^{13} \mathrm{C}$ NMR $\left(\mathrm{CDCl}_{3}\right) \delta-1.6,-1.5,14.1,17.3,18.2,23.0,25.9,26.1$, $30.4,31.0,32.4,58.0,59.6,65.9,69.0,73.8,127.2,128.2,128.5,141.7$.

Preparation of aminal 10e. A solution of 9 e $(929 \mathrm{mg}, 2.20 \mathrm{mmol})$ in dry methanol $(36 \mathrm{~mL})$ containing palladium catalyst $(10 \% \mathrm{Pd} / \mathrm{C}, 186 \mathrm{mg})$ was stirred at room temperature under hydrogen atmosphere. After $48 \mathrm{~h}$, catalyst was removed by filtration through celite and the solvent removed in vacuo. The residue was purified by flash chromatography (AcOEt/MeCN/NH $4 \mathrm{OH}, 237 / 12 / 1)$ to provide 10e as a pale yellow amorphous solid (633 mg, $68 \%)$.

$(1 S, \quad 5 S)$ 8-[2-(tert-Butyl-dimethyl-silanyloxy)-(1R)-phenyl-ethyl]-(7R)-pyridin-2-yl-6,8diazabicyclo[3.2.1]octane 10e. amorphous. $[\alpha] \mathrm{D}=-31\left(c\right.$ 1.4, $\left.\mathrm{CH}_{2} \mathrm{Cl}_{2}\right)$; IR (neat) 3299, 2929, 2857, $1592 \mathrm{~cm}^{-1}$; MS m/z $424\left(\mathrm{MH}^{+}\right) ;{ }^{1} \mathrm{H}$ NMR $\left(\mathrm{CDCl}_{3}\right) \delta-0.15(\mathrm{~s}, 3 \mathrm{H}),-0.13(\mathrm{~s}, 3 \mathrm{H})$, 0.78 (s, 9H), 1.51-1.94 (m, 7H), 3.21 (br. s, 1H), 3.38 (t, $J=5.5 \mathrm{~Hz}, 1 \mathrm{H}), 3.44$ (dd, $J=10.2$, $5.2 \mathrm{~Hz}, 1 \mathrm{H}), 3.80$ (dd, $J=10.2,5.7 \mathrm{~Hz}, 1 \mathrm{H}), 4.15$ (br.s, $1 \mathrm{H}), 4.98$ (br.s, $1 \mathrm{H}), 6.61$ (d, $J=6.8$ $\mathrm{Hz}, 1 \mathrm{H}), 6.92-7.06(\mathrm{~m}, 4 \mathrm{H}), 7.10(\mathrm{dd}, J=7.5,4.9,1 \mathrm{H}), 7.46$ (td, $J=7.5,1.8 \mathrm{~Hz}, 1 \mathrm{H}), 8.51$ (d, $J=4.9 \mathrm{~Hz}, 1 \mathrm{H}) ;{ }^{13} \mathrm{C} \mathrm{NMR}\left(\mathrm{CDCl}_{3}\right) \delta-1.5,-1.4,17.7,18.1,25.8,31.9,33.0,65.1,65.2,66.2$, $69.2,76.0,121.4,121.8,126.7,127.7,128.2,136.0,141.5,147.8,162.3$.

General procedure for the preparation of diamines $8 \mathrm{~b}, \mathbf{8 d}, \mathbf{8 f}$. The preparation of diamine 8d is representative. $\mathrm{LiAlH}_{4}(445 \mathrm{mg}, 11.7 \mathrm{mmol})$ was carefully added at room temperature to a solution of aminal 11d $(1.32 \mathrm{~g}, 2.93 \mathrm{mmol})$ in $\mathrm{Et}_{2} \mathrm{O}(50 \mathrm{~mL})$ under argon. The reaction mixture was stirred for $24 \mathrm{~h}$ at reflux, then allowed to cool and treated at room temperature 
successively with aqueous $\mathrm{NaOH}(1 \mathrm{~N}, 2.64 \mathrm{~mL})$ and $\mathrm{H}_{2} \mathrm{O}(3.96 \mathrm{~mL})$. After filtration through celite and washing the residue several times with $\mathrm{Et}_{2} \mathrm{O}$, the organic layer was concentrated in vacuo. The residue was purified by flash chromatography (AcOEt/MeCN/NH $4 \mathrm{OH}, 237: 12: 1$ ) to provide $8 \mathbf{d}$ as a colorless oil (488 $\mathrm{mg}, 49 \%)$.

$(2 R)-[(2 R)-(2-M e t h o x y-p h e n y l)-a z e p a n-3 S$-ylamino]-2-phenyl-ethanol 8d. Oil. $[\alpha] \mathrm{D}=-62$ (c 1.4, $\mathrm{CH}_{2} \mathrm{Cl}_{2}$ ); IR (neat) 3407, 2928, $1600 \mathrm{~cm}^{-1} ; \mathrm{MS} \mathrm{m} / z 341\left(\mathrm{MH}^{+}\right) ;{ }^{1} \mathrm{H} \mathrm{NMR}\left(\mathrm{CDCl}_{3}\right) \delta$ 1.42-1.92 (m, 9H), $2.79(\mathrm{~m}, 1 \mathrm{H}), 2.95(\mathrm{~m}, 1 \mathrm{H}), 3.15(\mathrm{~m}, 1 \mathrm{H}), 3.33(\mathrm{dd}, J=11.6,4.2 \mathrm{~Hz}, 1 \mathrm{H})$, $3.84(\mathrm{~d}, J=7.6 \mathrm{~Hz}, 1 \mathrm{H}), 3.86(\mathrm{~s}, 3 \mathrm{H}), 6.91(\mathrm{~d}, J=7.8 \mathrm{~Hz}, 1 \mathrm{H}), 6.98(\mathrm{td}, J=7.8-1.4 \mathrm{~Hz}, 1 \mathrm{H})$, 7.08-7.30 (m, 6H), $7.34(\mathrm{dd}, J=7.5,1.4 \mathrm{~Hz}, 1 \mathrm{H}) ;{ }^{13} \mathrm{C} \mathrm{NMR}\left(\mathrm{CDCl}_{3}\right) \delta 21.9,31.0,34.5,49.8$, $55.5,63.2,63.6,66.0,110.9,121.1,127.0,127.3,128.1,128.3,128.5,133.6,142.5,156.7$. HRMS calcd for $\mathrm{C}_{21} \mathrm{H}_{29} \mathrm{~N}_{2} \mathrm{O}_{2}\left(\mathrm{MH}^{+}\right): 341.2229$, found : 341.2231 .

2-[(2R)-Butyl-azepan-(3S)-ylamino]-(2R)-phenyl-ethanol $8 \mathbf{b}$ was prepared from compound 11b according to the previous procedure, although $\mathrm{Et}_{2} \mathrm{O}$ was replaced by $i-\operatorname{Pr}_{2} \mathrm{O}$. Pure $8 \mathbf{b}$ was obtained without any purification, as a colorless oil $(833 \mathrm{mg}, 98 \%) .[\alpha] \mathrm{D}=-17(c$ 1.0, $\mathrm{MeOH})$; IR (neat) 3425, $1636 \mathrm{~cm}^{-1} ; \mathrm{MS} m / z 291\left(\mathrm{MH}^{+}\right) ;{ }^{1} \mathrm{H}$ NMR $\left(\mathrm{CDCl}_{3}\right) \delta 0.84(\mathrm{t}, J=6.4$ Hz, 3H), 1.12-1.63 (m, 12H), 2.14 (br.s, 3H), 2.34 (m, 1H), 2.47 (m, 1H), $2.54(\mathrm{~m}, 1 \mathrm{H}), 2.97$ (dt, $J=13.2,4.0 \mathrm{~Hz}, 1 \mathrm{H}), 3.40$ (dd, $J=10.6,7.6 \mathrm{~Hz}, 1 \mathrm{H}), 3.58$ (dd, $J=10.6,4.7 \mathrm{~Hz}, 1 \mathrm{H}), 3.70$ $(\mathrm{dd}, J=7.6,4.8 \mathrm{~Hz}, 1 \mathrm{H}), 7.05-7.33(\mathrm{~m}, 5 \mathrm{H}) ;{ }^{13} \mathrm{C} \mathrm{NMR}\left(\mathrm{CDCl}_{3}\right) \delta 14.5,22.1,23.2,29.4,32.0$, $32.3,34.4,49.0,62.2,63.3,66.6,127.4,127.8,128.9,142.8$.

(2R)-[(3S)-(2-Hydroxy-(1R)-phenyl-ethylamino)-azepan-2-yl]-phenol $8 \mathrm{f}$ was prepared from compound 11d according to previous procedure, although $\mathrm{Et}_{2} \mathrm{O}$ was replaced by $i-\operatorname{Pr}_{2} \mathrm{O}$. The residue was purified by flash chromatography (AcOEt/MeCN/ $\mathrm{NH}_{4} \mathrm{OH}, 237: 12: 1$ ) to provide compound $8 \mathrm{f}$ as a colorless oil (478 mg, 50\%). [ $\alpha] \mathrm{D}=-17\left(c 1.1, \mathrm{CH}_{2} \mathrm{Cl}_{2}\right)$; IR (neat) 3301, 2928, $1732 \mathrm{~cm}^{-1}$; MS m/z $327\left(\mathrm{MH}^{+}\right) ;{ }^{1} \mathrm{H}$ NMR $\left(\mathrm{CDCl}_{3}\right) \delta 1.50-1.92(\mathrm{~m}, 9 \mathrm{H}), 2.78(\mathrm{~m}$, 1H), $2.94(\mathrm{~m}, 1 \mathrm{H}), 3.12(\mathrm{dt}, J=13.7,4.3 \mathrm{~Hz}, 1 \mathrm{H}), 3.33(\mathrm{dd}, J=10.5,6.7 \mathrm{~Hz}, 1 \mathrm{H}), 3.41-3.52$ 
(m, 2H), $3.72(\mathrm{~d}, J=7.9 \mathrm{~Hz}, 1 \mathrm{H}), 6.85(\mathrm{td}, J=7.8,1.2 \mathrm{~Hz}, 1 \mathrm{H}), 6.92(\mathrm{dd}, J=7.8,1.1 \mathrm{~Hz}, 1 \mathrm{H})$, 7.11-7.35 (m, 7H); ${ }^{13} \mathrm{C}$ NMR $\left(\mathrm{CDCl}_{3}\right) \delta 21.9,31.0,34.6,49.8,55.5,63.2,63.6,66.0,110.9$, $121.1,127.0,127.3,128.0,128.2,128.5,133.7,142.6,156.8$.

\section{General procedure for the synthesis of 2,3-diamines 3a,b,d,f.}

The preparation of diamine 3d is representative. Stirring under hydrogen atmosphere of compound 8d (645 mg, $1.90 \mathrm{mmol})$ in methanol $(30 \mathrm{~mL})$ in the presence of aqueous $\mathrm{HCl}(\mathrm{pH}$ 2) and $10 \% \mathrm{Pd} / \mathrm{C}(129 \mathrm{mg})$ for $48 \mathrm{~h}$ at room temperature afforded a compound, which after filtration and concentration, was dissolved in ether and extracted three times with an aqueous $2 \mathrm{~N} \mathrm{HCl}$ solution. Aqueous layers were made basic $(\mathrm{NaOH} 6 \mathrm{~N})$ and then extracted with AcOEt. The organic layer were dried $\left(\mathrm{Na}_{2} \mathrm{SO}_{4}\right)$ and concentrated to provide $\mathbf{3 d}$ as a colorless oil (397 mg, 95\%).

(2R)-(2-Methoxyphenyl)-azepan-(3S)-ylamine 3d. Oil. [ $\alpha] \mathrm{D}=-42$ (c 1.1, $\left.\mathrm{CH}_{2} \mathrm{Cl}_{2}\right)$; IR (neat) 3355, 2928, $1640 \mathrm{~cm}^{-1}$; MS $m / z 221\left(\mathrm{MH}^{+}\right) ;{ }^{1} \mathrm{H}$ NMR $\left(\mathrm{CDCl}_{3}\right) \delta 1.42-2.08(\mathrm{~m}, 8 \mathrm{H})$, $2.86(\mathrm{~m}, 2 \mathrm{H}), 3.14(\mathrm{~m}, 1 \mathrm{H}), 3.72(\mathrm{~d}, J=9.4 \mathrm{~Hz}, 1 \mathrm{H}), 3.83(\mathrm{~s}, 3 \mathrm{H}), 6.88(\mathrm{~d}, J=7.5 \mathrm{~Hz}, 1 \mathrm{H})$, $6.95(\mathrm{td}, J=7.5,1.4 \mathrm{~Hz}, 1 \mathrm{H}), 7.22(\mathrm{td}, J=7.5,1.5 \mathrm{~Hz}, 1 \mathrm{H}), 7.34(\mathrm{dd}, J=7.5,1.4 \mathrm{~Hz}, 1 \mathrm{H}) ;{ }^{13} \mathrm{C}$ NMR $\left(\mathrm{CDCl}_{3}\right) \delta 22.0,30.1,36.3,49.1,55.5,58.0,66.1,110.8,121.1,127.9,128.9,132.5$, 156.9; HRMS calcd for $\mathrm{C}_{13} \mathrm{H}_{21} \mathrm{~N}_{2} \mathrm{O}\left(\mathrm{MH}^{+}\right)$221.1654, found 221.1654.

(2R)-Butyl-azepan-(3S)-ylamine $3 \mathbf{b}$ was prepared from compound $\mathbf{8 b}$ (663 $\mathrm{mg}, 95 \%)$. It proved to be air sensitive and was characterized in its bis hydrochloride form (prepared after standing in an $\mathrm{HCl}$ atmosphere for $0.5 \mathrm{~h})$. [ $\alpha] \mathrm{D}=+3(c 1.0, \mathrm{MeOH})$; IR (neat) $3418 \mathrm{~cm}^{-1}$; MS (base) $m / z 171\left(\mathrm{MH}^{+}\right) ;{ }^{1} \mathrm{H}$ NMR $\left(\mathrm{D}_{2} \mathrm{O}\right.$, hydrochloride) $\delta 0.72(\mathrm{t}, J=6.9 \mathrm{~Hz}, 3 \mathrm{H}), 1.03-2.00$ (m, 15H), $3.08(\mathrm{~m} \mathrm{1H}), 3.24(\mathrm{~m}, 1 \mathrm{H}), 3.35(\mathrm{dt}, J=11.9,3.8 \mathrm{~Hz}, 1 \mathrm{H}), 3.48(\mathrm{dt}, J=11.9,4.1$ $\mathrm{Hz}, 1 \mathrm{H}) ;{ }^{13} \mathrm{C} \mathrm{NMR}\left(\mathrm{CDCl}_{3}\right) \delta 14.5,22.3,22.7,26.3,27.4,31.4,31.8,47.9,54.0,61.8$; HRMS calcd for $\mathrm{C}_{10} \mathrm{H}_{23} \mathrm{~N}_{2}\left(\mathrm{MH}^{+}\right)$171.1861, found 170.1863 . 
2-[(3S)-Amino-azepan-(2R)-yl]-phenol 3f (Oil, $272 \mathrm{mg}, 90 \%)$. [ $\alpha] \mathrm{D}=-85\left(c\right.$ 1.0, $\left.\mathrm{CH}_{2} \mathrm{Cl}_{2}\right)$; IR (neat): 3352, 2929, $1588 \mathrm{~cm}^{-1}$; MS m/z $207\left(\mathrm{MH}^{+}\right) ;{ }^{1} \mathrm{H}$ NMR $\left(\mathrm{CDCl}_{3}\right) \delta 1.60-1.90(\mathrm{~m}$, 6H), 2.80 (td, $J=13.4,3.4 \mathrm{~Hz}, 1 \mathrm{H}), 3.03-3.19(\mathrm{~m}, 2 \mathrm{H}), 3.50$ (d, $J=8.7 \mathrm{~Hz}, 1 \mathrm{H}), 3.60$ (br.s, $2 \mathrm{H}), 6.81(\mathrm{td}, J=7.8,1.1 \mathrm{~Hz}, 1 \mathrm{H}), 6.87(\mathrm{dd}, J=8.8,1.0 \mathrm{~Hz}, 1 \mathrm{H}), 7.07-7.20(\mathrm{~m}, 2 \mathrm{H}) ;{ }^{13} \mathrm{C}$ NMR $\left(\mathrm{CDCl}_{3}\right) \delta 22.2,28.4,34.5,47.6,56.5,71.5,117.5,119.2,128.1,128.8,156.4$; HRMS calcd for $\mathrm{C}_{12} \mathrm{H}_{19} \mathrm{~N}_{2} \mathrm{O}\left(\mathrm{MH}^{+}\right)$207.1497, found 207.1499.

\section{General Procedure for the preparation of trisubstituted azepanes 14a-c.}

The preparation of diamine $\mathbf{1 4} \mathbf{a}$ is representative. An ethereal solution of methyl magnesium bromide ( $\left.3 \mathrm{M} / \mathrm{Et}_{2} \mathrm{O}, 2.71 \mathrm{~mL}, 8.12 \mathrm{mmol}\right)$ was added at room temperature to a solution of aminal 11a (976 mg, $2.31 \mathrm{mmol})$ in ether $(40 \mathrm{~mL})$ under argon. The reaction mixture was stirred for $3 \mathrm{~h}$ at reflux. The reaction mixture was cautiously diluted with a saturated, aqueous solution of $\mathrm{NH}_{4} \mathrm{Cl}$, extracted with $\mathrm{CH}_{2} \mathrm{Cl}_{2}(2 \mathrm{x})$, and the combined organic layers were dried $\left(\mathrm{MgSO}_{4}\right)$. The residue was purified by flash chromatography (AcOEt/MeCN/ $\mathrm{NH}_{4} \mathrm{OH}$, $237 / 12 / 1)$ to afford $14 \mathbf{a}$ as a yellow oil (577 $\mathrm{mg}, 57 \%)$.

\section{[2-(tert-Butyl-dimethyl-silanyloxy)-(1R)-phenyl-ethyl]-(7R-methyl-(2R)-phenyl-azepan-}

(3S)-yl)-amine 14a. Oil. [ $\alpha$ ] $\mathrm{D}=-16\left(c 0.6, \mathrm{CH}_{2} \mathrm{Cl}_{2}\right)$; IR (neat) 3362, $2927 \mathrm{~cm}^{-1} ; \mathrm{MS} \mathrm{m} / z 439$ $\left(\mathrm{MH}^{+}\right) ;{ }^{1} \mathrm{H}$ NMR $\left(\mathrm{CDCl}_{3}\right) \delta-0.21(\mathrm{~s}, 6 \mathrm{H}), 0.70(\mathrm{~s}, 9 \mathrm{H}), 0.97(\mathrm{~d}, J=6.4 \mathrm{~Hz}, 3 \mathrm{H}), 1.10-1.85$ (m, 8H), $2.90(\mathrm{~m}, 1 \mathrm{H}), 3.01(\mathrm{~m}, 1 \mathrm{H}), 3.23-3.38(\mathrm{~m}, 3 \mathrm{H}), 3.82$ (d, $J=8.6 \mathrm{~Hz}, 1 \mathrm{H}), 7.10-7.35$

$(\mathrm{m}, 10 \mathrm{H}) ;{ }^{13} \mathrm{C} \mathrm{NMR}\left(\mathrm{CDCl}_{3}\right) \delta-1.4,18.4,23.3,23.4,26.0,36.6,37.6,49.5,62.2,62.8,63.6$, $68.0,127.0,127.1,127.5,127.8,128.1,128.8,142.8,145.1$.

\section{[2-(tert-Butyl-dimethyl-silanyloxy)-1 $R$-phenyl-ethyl]-(7S-isobutyl-(2R)-phenyl-azepan-} (3S)-yl)-amine 14b (Oil, $931 \mathrm{mg}, 84 \%)$. [ $\alpha] \mathrm{D}=-16\left(c \mathrm{0} .8, \mathrm{CH}_{2} \mathrm{Cl}_{2}\right)$; IR (neat) 3356, 2928, $1600 \mathrm{~cm}^{-1}$; MS m/z $481\left(\mathrm{MH}^{+}\right) ;{ }^{1} \mathrm{H}$ NMR $\left(\mathrm{CDCl}_{3}\right) \delta-0.12(\mathrm{~s}, 6 \mathrm{H}), 0.72(\mathrm{~d}, J=6.2 \mathrm{~Hz}, 6 \mathrm{H})$, $0.80(\mathrm{~s}, 9 \mathrm{H}), 1.00-1.90(\mathrm{~m}, 9 \mathrm{H}), 2.89(\mathrm{~m}, 1 \mathrm{H}), 3.0(\mathrm{td}, J=8.7,3.6 \mathrm{~Hz}, 1 \mathrm{H}), 3.30-3.46(\mathrm{~m}$, $3 \mathrm{H}), 3.75(\mathrm{~d}, J=8.7 \mathrm{~Hz}, 1 \mathrm{H}), 7.18-7.40(\mathrm{~m}, 10 \mathrm{H}) ;{ }^{13} \mathrm{C} \mathrm{NMR}\left(\mathrm{CDCl}_{3}\right) \delta-1.5,18.4,22.3$, 
$23.2,23.5,24.6,26.0,36.3,36.6,46.4,51.7,62.4,63.0,63.7,68.0,127.0,127.2,127.7$, $127.8,128.1,128.7,142.8,144.8$.

[(7S)-Benzyl-(2R)-phenyl-azepan-(3S)-yl]-[2-(tert-butyl-dimethyl-silanyloxy)-(1R)phenyl-ethyl]-amine 14c (Oil, $1.13 \mathrm{~g}, 95 \%) .[\alpha] \mathrm{D}=+15\left(c 1.1, \mathrm{CH}_{2} \mathrm{Cl}_{2}\right.$ ); IR (neat) 3415, $2926 \mathrm{~cm}^{-1} ; \mathrm{MS} \mathrm{m} / z 515\left(\mathrm{MH}^{+}\right) ;{ }^{1} \mathrm{H}$ NMR $\left(\mathrm{CDCl}_{3}\right) \delta-0.20(\mathrm{~s}, 6 \mathrm{H}), 0.75(\mathrm{~s}, 9 \mathrm{H}), 1.20-1.90(\mathrm{~m}$, 8H), $2.53(\mathrm{dd}, J=13.3,8.2 \mathrm{~Hz}, 1 \mathrm{H}), 2.62(\mathrm{dd}, J=13.3,5.6 \mathrm{~Hz}, 1 \mathrm{H}), 2.90(\mathrm{~m}, 1 \mathrm{H}), 3.03$ (m, 1H), 3.27 (dd, $J=13.1,7.5 \mathrm{~Hz}, 1 \mathrm{H}), 3.38(\mathrm{dd}, J=13.1,7.5 \mathrm{~Hz}, 1 \mathrm{H}), 3.76(\mathrm{~d}, J=8.5 \mathrm{~Hz}, 1 \mathrm{H})$, 6.87-7.40 (m, 15H); ${ }^{13} \mathrm{C}$ NMR $\left(\mathrm{CDCl}_{3}\right) \delta$-1.4, 18.4, 23.2, 26.0, 35.5, 36.9, 43.4, 56.3, 62.7, $62.8,63.5,68.0,127.0,127.1,127.6,127.8,128.1,128.3,128.7,129.3,139.5,142.8,144.2$.

\section{[2-(tert-Butyl-dimethyl-silanyloxy)-(1R)-phenyl-ethyl]-(7R-methyl-2-pyridin-(2R)-yl-} azepan-(3S)-yl)-amine 14d (Oil, $739 \mathrm{mg}, 69 \%)$. [ $\alpha]_{\mathrm{D}}=-15\left(c 0.8, \mathrm{CH}_{2} \mathrm{Cl}_{2}\right)$; IR (neat) 3424, 2929, $\mathrm{cm}^{-1} ; \mathrm{MS} \mathrm{m} / z 440\left(\mathrm{MH}^{+}\right) ;{ }^{1} \mathrm{H}$ NMR $\left(\mathrm{CDCl}_{3}\right) \delta-0.15(\mathrm{~s}, 3 \mathrm{H}),-0.13(\mathrm{~s}, 3 \mathrm{H}), 0.80(\mathrm{~s}$, 9H), 1.10-1.50 (m, 6H), 1.30 (d, $J=6.7 \mathrm{~Hz}, 3 \mathrm{H}), 2.05$ (br.s, 2H), 2.75 (m, 2H), $3.54(\mathrm{~m}, 1 \mathrm{H})$, $3.54(\mathrm{~d}, J=5.0 \mathrm{~Hz}, 1 \mathrm{H}), 3.67(\mathrm{dd}, J=7.6,4.3 \mathrm{~Hz}, 1 \mathrm{H}), 3.78(\mathrm{dd}, J=13.5,7.6 \mathrm{~Hz}, 1 \mathrm{H}), 7.01$ $(\mathrm{dd}, J=6.6,5.0 \mathrm{~Hz}, 1 \mathrm{H}), 7.10-7.33(\mathrm{~m}, 6 \mathrm{H}), 7.51(\mathrm{td}, J=8.2,1.7 \mathrm{~Hz}, 1 \mathrm{H}), 8.42$ (br.d, $J=5.0$ $\mathrm{Hz}, 1 \mathrm{H}) ;{ }^{13} \mathrm{C} \mathrm{NMR}\left(\mathrm{CDCl}_{3}\right) \delta-1.3,18.7,20.1,23.5,26.1,30.3,30.7,58.7,58.9,59.7,64.9$, $68.9,121.2,121.8,127.2,127.9,128.2,128.4,136.4,142.7,149.1,167.8$.

\section{[2-(tert-Butyl-dimethyl-silanyloxy)-(1R)-phenyl-ethyl]-(7S-isobutyl-2-pyridin-(2R)-yl-} azepan-(3S)-yl)-amine 14e (Oil, $822 \mathrm{mg}, 70 \%)$. [ $\alpha] \mathrm{D}=-12\left(c 0.8, \mathrm{CH}_{2} \mathrm{Cl}_{2}\right)$; IR (neat) 3412, 2928, 1468, $1434 \mathrm{~cm}^{-1}$; MS m/z $482\left(\mathrm{MH}^{+}\right) ;{ }^{1} \mathrm{H}$ NMR $\left(\mathrm{CDCl}_{3}\right) \delta-0.15(\mathrm{~s}, 3 \mathrm{H}),-0.13(\mathrm{~s}, 3 \mathrm{H})$, $0.75(\mathrm{~s}, 9 \mathrm{H}), 0.82(\mathrm{~m}, 7 \mathrm{H}), 0.90-1.60(\mathrm{~m}, 6 \mathrm{H}), 1.51(\mathrm{t}, J=6.5 \mathrm{~Hz}, 2 \mathrm{H}), 2.17$ (br.s, 2H), 2.73 (m, 2H), $3.55(\mathrm{~m}, 1 \mathrm{H}), 3.55$ (d, $J=5.8 \mathrm{~Hz}, 1 \mathrm{H}), 3.67$ (m, 2H), 6.99 (ddd, $J=7.5,4.5,1.3 \mathrm{~Hz}$, $1 \mathrm{H}), 7.10-7.30(\mathrm{~m}, 6 \mathrm{H}), 7.49(\mathrm{td}, J=7.5,1.3 \mathrm{~Hz}, 1 \mathrm{H}), 8.41(\mathrm{~d}, J=4.5 \mathrm{~Hz}, 1 \mathrm{H}) ;{ }^{13} \mathrm{C} \mathrm{NMR}$ 
$\left(\mathrm{CDCl}_{3}\right) \delta 1.3,18.5,20.0,23.0,25.0,26.1,30.4,30.6,47.2,59.6,59.7,62.6,64.0,68.8$, $121.7,122.0,127.2,127.9,128.1,136.1,142.6,149.0,166.3$.

[(7S)-Benzyl-2-pyridin-(2R)-yl-azepan-(3S)-yl]-[2-(tert-butyl-dimethyl-silanyloxy)-(1R)phenyl-ethyl]-amine $14 f(\mathrm{Oil}, 817 \mathrm{mg}, 65 \%)$. [ $\alpha]_{\mathrm{D}}=-11\left(c \mathrm{c} 1.1, \mathrm{CH}_{2} \mathrm{Cl}_{2}\right)$; IR (neat) 3390, 3028, $2952 \mathrm{~cm}^{-1}$; MS m/z $516\left(\mathrm{MH}^{+}\right) ;{ }^{1} \mathrm{H}$ NMR $\left(\mathrm{CDCl}_{3}\right) \delta-0.20(\mathrm{~s}, 3 \mathrm{H}),-0.15(\mathrm{~s}, 3 \mathrm{H}), 0.75$ (s, 9H), 1.00-1.40 (m, 8H), $2.60(\mathrm{~m}, 2 \mathrm{H}), 2.79(\mathrm{dd}, J=13.6,8.6 \mathrm{~Hz}, 1 \mathrm{H}), 2.99$ (dd, $J=13.6$, $5.7 \mathrm{~Hz}, 1 \mathrm{H}), 3.24(\mathrm{dd}, J=6.6,4.5 \mathrm{~Hz}, 1 \mathrm{H}), 3.30(\mathrm{~m}, 2 \mathrm{H}), 3.89(\mathrm{dd}, J=8.6,5.7 \mathrm{~Hz}, 1 \mathrm{H}), 6.97$ (ddd, $J=7.6,4.8,1.5 \mathrm{~Hz}, 1 \mathrm{H}), 7.04-7.27$ (m, 11H), 7.44 (td, $J=7.6,1.5 \mathrm{~Hz}, 1 \mathrm{H}), 8.34$ (ddd, $J$ $=4.8,1.7,0.7 \mathrm{~Hz}, 1 \mathrm{H}) ;{ }^{13} \mathrm{C} \mathrm{NMR}\left(\mathrm{CDCl}_{3}\right) \delta-1.2,18.4,20.0,26.0,30.2,30.5,44.1,59.3$, $59.6,63.7,65.6,68.4,121.1,121.9,126.3,127.0,127.5,127.9,128.0,128.4,128.5,129.5$, $136.3,139.0,142.7,148.8,164.9$.

\section{General Procedure for the preparation of trisubstituted azepanes 4a-c}

The preparation of diamine $4 \mathbf{a}$ is representative. $\mathrm{BF}_{3} . \mathrm{Et}_{2} \mathrm{O}(809 \mu \mathrm{L}, 6.6 \mathrm{mmol})$ was added to a solution of azepane 14a $(577 \mathrm{mg}, 1.3 \mathrm{mmol})$ in $\mathrm{CHCl}_{3}(15 \mathrm{~mL})$ under argon. The reaction mixture was stirred $15 \mathrm{~h}$ at reflux, allowed to cool and treated with an aqueous saturated $\mathrm{NaHCO}_{3}$ solution. The organic layer were separated, dried on $\mathrm{MgSO}_{4}$ and the solvent was evaporated. The crude product was then submitted to hydrogenolysis in methanol $(15 \mathrm{~mL})$ under $\mathrm{H}_{2}$ atmosphere in the presence of $\mathrm{HCl}(\mathrm{pH} 2)$ and $10 \% \mathrm{Pd} / \mathrm{C}(76 \mathrm{mg})$ for $48 \mathrm{~h}$, to afford a compound, which after filtration and concentration, was dissolved in ether and extracted three times with aqueous $\mathrm{HCl}$ solution. Aqueous layers were made basic $(6 \mathrm{~N} \mathrm{NaOH})$ and then extracted with AcOEt. The organic layer was dried $\left(\mathrm{Na}_{2} \mathrm{SO}_{4}\right)$ and concentrated to give 4a as a colorless oil (224 $\mathrm{mg}, 70 \%)$.

(7R)-Methyl-(2R)-phenyl-azepan-(3S)-ylamine 4a. Oil. [ $\alpha] \mathrm{D}=-18\left(c \quad 0.8, \mathrm{CH}_{2} \mathrm{Cl}_{2}\right)$; IR (neat) 3363, 2924, 1644, $1600 \mathrm{~cm}^{-1}$; MS $m / z 205\left(\mathrm{MH}^{+}\right) ;{ }^{1} \mathrm{H}$ NMR $\left(\mathrm{CDCl}_{3}\right) \delta 1.08(\mathrm{~d}, J=6.3$ $\mathrm{Hz}, 3 \mathrm{H}), 1.17-2.15(\mathrm{~m}, 9 \mathrm{H}), 3.05-3.10(\mathrm{~m}, 2 \mathrm{H}), 3.55$ (d, $J=9.5 \mathrm{~Hz}, 1 \mathrm{H}), 7.17-7.40(\mathrm{~m}, 5 \mathrm{H})$; 
${ }^{13} \mathrm{C} \mathrm{NMR}\left(\mathrm{CDCl}_{3}\right) \delta 23.5,37.2,39.2,50.6,58.0,63.5,127.3,129.0,145.1$; HRMS (CI, $\mathrm{MH}^{+}$) calcd for $\mathrm{C}_{13} \mathrm{H}_{21} \mathrm{~N}_{2}$ 205.1705, found 205.1707.

(7S)-Isobutyl-(2R)-phenyl-azepan-(3S)-ylamine 4b (77\% yield, Oil). [ $\alpha]_{\mathrm{D}}=-20$ (c 0.9 , $\left.\mathrm{CHCl}_{3}\right)$; IR (neat) 3367, 2951, 1666, $1602 \mathrm{~cm}^{-1} ; \mathrm{MS} \mathrm{m} / z 247\left(\mathrm{MH}^{+}\right) ;{ }^{1} \mathrm{H}$ NMR $\left(\mathrm{CDCl}_{3}\right) \delta$ $0.78(\mathrm{~d}, J=6.3 \mathrm{~Hz}, 3 \mathrm{H}), 0.82(\mathrm{~d}, J=6.3 \mathrm{~Hz}, 3 \mathrm{H}), 1.12-1.80(\mathrm{~m}, 10 \mathrm{H}), 1.95(\mathrm{~m}, 1 \mathrm{H}), 2.12(\mathrm{~m}$, 1H), 2.99 (m, 1H), 3.10 (td, $J=9.5,4.4 \mathrm{~Hz}, 1 \mathrm{H}), 3.49$ (d, $J=9.5 \mathrm{~Hz}, 1 \mathrm{H}), 7.20-7.38$ (m, 5H); ${ }^{13} \mathrm{C} \mathrm{NMR}\left(\mathrm{CDCl}_{3}\right) \delta 22.3,23.0,23.1,24.7,35.6,39.3,46.1,52.7,57.8,63.1,127.3,128.9$, 144.7; HRMS (CI, $\mathrm{MH}^{+}$) calcd for $\mathrm{C}_{16} \mathrm{H}_{27} \mathrm{~N}_{2}$ 247.2174, found 247.2174.

(7S)-Benzyl-(2R)-phenyl-azepan-(3S)-ylamine 4c (81\%, oil). [ $\alpha] \mathrm{D}=+67\left(\right.$ c 1.0, $\left.\mathrm{CHCl}_{3}\right)$; IR (neat) 3359, 3025, 2926, 1673, $1601 \mathrm{~cm}^{-1}$; MS m/z $281\left(\mathrm{MH}^{+}\right) ;{ }^{1} \mathrm{H}$ NMR $\left(\mathrm{CDCl}_{3}\right) \delta 1.17-$ $2.17(\mathrm{~m}, 9 \mathrm{H}), 2.63(\mathrm{dd}, J=13.6,8.3 \mathrm{~Hz}, 1 \mathrm{H}), 2.70(\mathrm{dd}, J=13.6,6.0 \mathrm{~Hz}, 1 \mathrm{H}), 3.06(\mathrm{td}, \mathrm{J}=9.5$, $4.5 \mathrm{~Hz}, 1 \mathrm{H}), 3.18(\mathrm{~m}, 1 \mathrm{H}), 3.59(\mathrm{~d}, J=9.5 \mathrm{~Hz}, 1 \mathrm{H}), 6.91-7.38(\mathrm{~m}, 10 \mathrm{H}) ;{ }^{13} \mathrm{C} \mathrm{NMR}\left(\mathrm{CDCl}_{3}\right) \delta$ 23.0, 35.2, 39.5, 43.3, 57.5, 58.3, 63.1, 126.0, 127.4, 128.3, 128.6, 128.9, 129.3, 139.6, 144.3; HRMS (CI, $\mathrm{MH}^{+}$) calcd for $\mathrm{C}_{19} \mathrm{H}_{25} \mathrm{~N}_{2} 281.2018$, found 281.2015.

Acknowledgment. S. C. wishes to thank the "Ligue Nationale contre le Cancer" for a grant.

Supporting Information Available : ${ }^{1} \mathrm{H}$ and ${ }^{13} \mathrm{C}$ NMR spectra of all new compounds. X-ray structure determination and crystal data for compound 15. This material is free of charge via the Internet at http://pubs.acs.org.

\section{References and notes}

(1) Lucet, D. ; Le Gall, T.; Mioskowski, C. Angew. Chem. Int. Ed. Engl. 1998, 37, 2581. 
(2) Chong, H.-S.; Garmestani, K.; Bryant, L. H.; Brechbiel, M. W. J. Org. Chem. 2001, 66, 7745 .

(3) a) Matecka, D.; Rothman, R. B.; Radesca, L.; De Costa, B. R.; Dersch, C. M.; Partilla, J. S.; Pert, A.; Glowa, J. R.; Wojnicki, H. E.; Rice, K. C. J. Med. Chem. 1996, 39, 4704; b) Hirokawa, Y.; Morie, T.; Yamazaki, H.; Yoshida, N.; Kato, S. Bioorg. Med. Chem. Lett. 1998, 8, 619; c) Zhao, S.; Freeman, J. P.; Bacon, C. L.; Fox, G. B.; O'Driscoll, E.; Foley, A. G.; Kelly, J.; Farrell, U.; Regan, C.; Mizsak, S. A.; Szmuszkovicz, J. Bioorg. Med. Chem. 1999, 7, 1647.

(4) Rezler, E. M.; Fenton, R. R.; Esdale, W. J.; McKeage, M. J.; Russell, P. J.; Hambley, T. W. J. Med. Chem. 1997, 40, 3508.

(5) a) Meyers, A. I.; Downing, S. V.; Weiser, M. J. J. Org. Chem. 2001, 66, 1413; b) Chong, H.-S.; Ganguly, B.; Broker, G. A.; Rogers, R. D.; Brechbiel, M. W. J. Chem. Soc., Perkin Trans. 1, 2002, 2080 and references herein; c) Kantorowski, E. J.; Kurth, M. J. Tetrahedron, 2000, 56, 4317; d) Evans, A. P.; Robinson, J. E. Org. Lett. 1999, 1, 1929.

(6) Cutri, S.; Bonin, M.; Micouin, L.; Froelich, O.; Quirion, J.-C.; Husson, H.-P. Tetrahedron Lett. 2000, 41, 1179.

(7) Bonin, M.; Grierson, D. S.; Royer, J.; Husson, H.-P.; Org. Synth., 1992, 70, 54.

(8) Froelich, O.; Desos, P.; Bonin, M.; Quirion, J.-C.; Husson, H.-P. J. Org. Chem. 1996, 61, 6700 .

(9) Corey, E. J.; Imwinkelried, R.; Pikul S.; Xiang, Y. B. J. Am. Chem. Soc. 1989, $111,5493$.

(10) Such reductive demethylation has been previously described on orthosubstituted methoxyphenols : Kimura, K.; Tanaka, M.; Iketani S.-I.; Shono, T. J. Org. Chem. 1987, 52, 836.

(11) a) For a general review on aminals preparation, see Pawlenko, S.; LangFugmann, S. In Houben-Weyl Methoden der Organische Chemie; Hageman, 
H. ; Klaman, D. Eds ; Georg Thieme Verlag : Stuttgart, 1992, E14a/3. Aminals as chiral auxiliary : b) Alexakis, A.; Mangeney, P.; Nelsen, N.; Tranchier, J. P.; Gosmini, R.; Raussou, S. Pure Appl. Chem. 1996, 68, 531; c) Jung, M.E.; Huang, A. Org. Lett. 2000, 2, 2659. Nucleophilic opening of oxazolidines: d) Bloch, R. Chem. Rev. 1998, 98, 1407; e) Enders, D.; Reinhold, U. Tetrahedron: Asymmetry, 1997, 8, 1895; f) Steinig, A. G.; Spero, D. M.; Org. Prep. Proc. Int., 2000, 32, 205.

(12) Stevens, R. V. Acc. Chem. Res.,1984, 17, 289.

\section{Table of Contents Graphic}

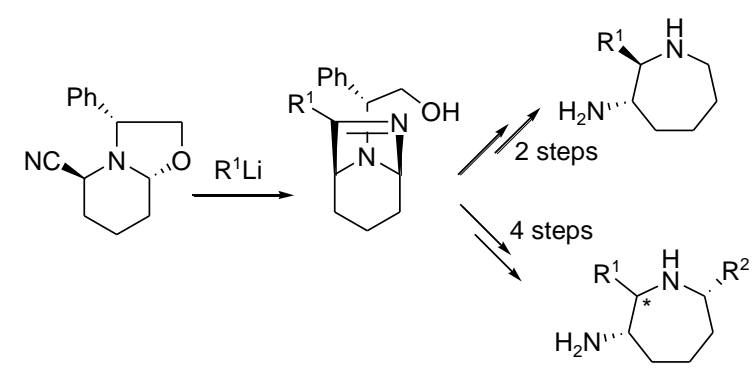

